\title{
What we have learned from the Rigopiano tragedy
}

\author{
Antonella Pescini, Paola D'alfonso \\ Red Cross, Abruzzo Regional Committee, L'Aquila, Italy
}

\begin{abstract}
On the afternoon of 18 January 2017, a major avalanche occurred on Gran Sasso d'Italia massif, destroying an hotel in Rigopiano, and killing twenty-nine people. A staff of psychologists trained in emergency psychology was involved to assist the families of the missing persons without ever abandoning them for eight consecutive days. Particular care was posed to identify an appropriate setting where families and psychologists could interact, favoring emotional containment and protection of intimacy in moments of intense pain. It was considered paramount that the team shared their intervention method, and that nobody operated individually, because this would support both families and members of the professional group. The long waiting for news about the missing persons' fate was a suspended time, during which the psychologists engaged in emotional rescue were called to express their empathy understanding and sharing silence. Once the bodies were retrieved, the recognition of corpses required the sharing of a common operational strategy to face lucid or contradictory communications, and the alternate feelings of disbelief, anger, or guilt. As a consequence of the Rigopiano tragedy, in the year 2018 the Abruzzo region wrote a new plan for maxi health emergencies, recognizing psychological suffering among the needs to be met in case of disaster.
\end{abstract}

\section{Foreward}

On the afternoon of 18 January 2017, a major avalanche occurred on Gran Sasso d'Italia massif, one of the mountains above Rigopiano, impacting and destroying the 4-star Hotel Rigopiano in Farindola, Abruzzo. The impact killed twenty-nine people and injured eleven others, making the avalanche the deadliest in Italy since the White Friday avalanches in 1916, and the deadliest avalanche in Europe since the Galtür avalanche in

\footnotetext{
Correspondence: Antonella Pescini, Red Cross, Abruzzo Regional Committee, L'Aquila, Italy.

E-mail: antopescini@hotmail.it

Key words: Rigopiano tragedy; emergency psychology.

Received for publication: 2 December 2019.

Accepted for publication: 13 December 2019.

This work is licensed under a Creative Commons Attribution 4.0 License (by-nc 4.0).

๑C Copyright: the Author(s), 2019

Licensee PAGEPress, Italy

Emergency Care Journal 2019; 15:8725

doi:10.4081/ecj.2019.8725
}

1999. Two causal factors for the avalanche of 2017 include a series of earthquakes that struck the region earlier in the day and the record snowfall which occurred for days prior to the avalanche.

A staff of psychologists trained in emergency psychology was involved to assist the families without ever abandoning them for the eight consecutive days it took to reach all the victims (see Table 1). They alternated in shifts but never neglected the sense of continuity in the support offered. The team formed at the time shared a method of intervention, and nobody operated individually. The role of each professional was to support the relatives of the missing, but at the same time to offer support to the other members of the group when needed.

\section{The psychology of emergency}

The psychology of emergency comprises professional interventions and research activities which deal with the consequences, on a psychological level, of disastrous events of the more varied nature that can affect individuals, groups, or communities as a whole. ${ }^{1-6}$ This discipline is quite recent: until the eighties, emergency interventions in case of a catastrophe took care mostly of health, sheltering and nutrition needs. ${ }^{7-10}$ Only gradually the rescue system acquired the objective of a complete psycho-physical wellbeing of the individual and became aware of the need to respond also to the emotional reactions of the victims.

In emergency contexts the psychological literature has produced a classic distinction of victims as:

- primary victims (those directly affected by the drama): the direct victims of disruptive and sudden events, regardless of the severity of the material damage suffered, and the witnesses of grossly damaging facts that threatened or compromised the survival of a human being;

- secondary victims (those with close ties to the primary victims); first among them the victims' close relatives;

- tertiary or vicarious victims (the rescuers and those who care for the primary victims for a professional reason): rescuers, volunteers and professionals, that in any capacity they have assisted the victims and the survivors;

- fourth level victims (subjects of neighboring areas, or of similar categories to the victims, that can potentially identify with them).

The presence of psychologists on the site of a catastrophe is useful to accompany and support the event processing, facilitating both rescue operations and the reconstruction of personal and relational balances once the most critical moments are overcome. ${ }^{11,12}$ The psychologists involved must be formed to operate in emergency contexts, in order to be able to perform many specific tasks: to protect family members from emotional disorganization and cognitive dissonance, to moderate community emotional contagion, to filter trigger questions, and when necessary, to make up for the absentee if she/he had roles of protection towards minors, supporting the transitions. In order to better carry out these tasks, it is important that psychologists have previously experienced the ability to work in emergency settings and in close collaboration with 
the police responsible for investigations and/or with the research coordinators entrusted with the task of collecting useful information. They can also play a role in facilitating communication between the needs of the relief apparatus and the mental functioning of the subjects involved. Having access to certain (verified) information and providing clarity on what is not yet known they may help people in great distress to keep analytical skills functioning and to maintain a sense of reality.

The intervention conducted by psychologists during the Rigopiano emergency was directed to the secondary victims, mostly family members of the missing.

\section{The scenario}

The event was both dramatic and very complex, mainly because the rescue machine proceeded very slowly as a consequence of the exceptional wave of frost that had hit the Central Apennines, especially in Abruzzo, leaving many municipalities and population centers isolated, and making most of the road network impractical.

Although the intervention scenario was the field where the hotel had been swept away by the avalanche, the relatives who came to the accident site were first welcomed at a reception point within the Penne hospital. Later on, given the weather conditions and the impracticality of the communication routes, they were moved, together with the psychologists, to the assembly hall of the Santo Spirito Civil Hospital of Pescara. The great media coverage of the event made professional associations decide to send their psychologists in support of the professional resources already active in the structure. In short, in a completely spontaneous manner, at the Santo Spirito Civil Hospital in Pescara a task force of psychologists was established, ready to make a contribution to the difficult moment of the Abruzzo Region.

The immediate identification of a reception site for the victims' families has an important strategic sense to favor psychological support, containing the inevitable emotional reactions that may arise in the long hours waiting for news on one's missing person. The environment of the assembly hall, in addition to favoring emotional containment, was wide enough to ensure that families could protect their intimacy in moments of intense pain. This also helped avoiding the risk of emotional contagion. Particular attention was given to reducing environmental stress factors (noise, overcrowding, cold) that could favor personal distress. In large emergencies, the importance of defining a place where the relatives of the missing can be conveyed is often underestimated, as if it were a secondary problem, but in our experience, we were confirmed that the appropriateness of the setting was a strength of our intervention.

\section{A time for waiting}

We are used to thinking of emergencies as a hectic moment in which many activities are carried out at the same time. In our case it was quite the contrary, since the support of the missing persons' relatives saw the psychologists engaged in a long and grueling waiting time. Hours and days went by expecting news from the rescuers carrying out the searches at the crash site. During the period of waiting, the entire family lives a suspended time, full of uncertainties, looking forward only to the moment when it will receive news on its dispersed dear one. To understand the emotional experience of those who find themselves in this condition, it is necessary to understand the meaning that the sudden and unexpected absence of one of its members has for each single-family unit, and within its specific family history. A sudden disappearance creates a fracture that hinders the process of detachment, effectively preventing the recovery of emotional investments made on the other. The uncertainty that characterizes this type of experience leads people to ask themselves a series of questions and to formulate a myriad of hypotheses that produce an oscillation of emotions: joy, in the hope of a reunion, but also anger towards the missing; feeling of helplessness and despair in front of the hypothesis of a definitive separation. This emotional state is often accompanied by sad thoughts associated with guilt for not having been able to prevent the situation in which they find themselves. Images from the past are recalled in which they could have behaved differently to avoid what was going to happen. The participant psychologists had to share these emotional dynamics of anxiety and sadness, aggression and guilt, at various times while waiting for the discovery of the many dead bodies.

Information on the rescue process was scanty and infrequent. The inability to get news on their missing dears health status, lead the people involved to confront a number of assumptions often going with their mind to what they feared most. Previous experiences, from readings and narratives, favored the creation of imaginary scenarios with the function of bridging the lack of information.

Moreover, it should be considered that in the face of emergency events, the scene often fills up with a diverse crowd of people made up of rescue technicians, law enforcement, health care, real or not welcome friends, curious people attracted by the clamor and mass media that tend to make a show out of suffering and pain.

To contain this as much as possible, the organization of the $\mathrm{S}$. Spirito Hospital of Pescara protected the relatives of the missing persons through a task force of law enforcement officers responsible for controlling who was authorized to enter the assembly hall. The goal of this protection was to discourage the proliferation of collective investigation into a very fragile environment. Indeed, in emergency scenarios, the spread of information often triggers public dynamics which lead to the instrumentalization of the second-

Table 1. Number and kind of interventions.

\begin{tabular}{ll}
\hline People affected by the collapse of the hotel & 40 \\
People who died & 29 \\
\hline Identification of corpses with psychological support & 29 \\
Rescued minors who received psychological support & 5 \\
\hline People addressed by psychological support & 120 people a day \\
& Overall the service addressed over 400 people \\
Emergency psychologists on the field & 35 emergency psychologists rotated on 12 hours shifts. 10/12 psychologists were present at a time \\
\hline
\end{tabular}


ary victims' emotions. The hidden risk is that uncontrolled voices, delirious interpretations, prejudices and data distortions may proliferate.

In these situations, it is of great importance to manage the flow of communications by entrusting to a single point of reference the task of announcing the progress of the research and the results of the same. Providing untrusted information, in an attempt to alleviate the pain of the people you care for, can intensify anger and psychological harm if the information given is found to be inaccurate. This is what happened in the case of the victim S. F., initially given as a survivor in a statement by the prefect of Pescara and then identified among the victims. The rekindling of hope sharpens the subsequent reactions of pain, turning it into intense anger. In the age of the internet it may become very difficult to contain the passage of information, to select truthful contents from fake news, and to highlight the importance of offering only verified information. The container area for relatives and emotions, also performed with the aim of facilitating truthful, ascertained communications among the rescuers and the relatives of the victims.

In the Rigopiano tragedy, the wait for the discovery of the missing, given the extremely difficult climatic conditions in which the rescuers operated, lasted about a week, the last missing person was recovered under the avalanche on January $25^{\text {th }}$.

The psychologists involved in the event assisted the families without ever abandoning them for the whole period, alternating in shifts but never neglecting the sense of continuity in the support offered. The team members shared their intervention method, and nobody operated individually. The main role of the psychologists was to support the relatives of the missing persons, but it soon became clear that it was also necessary to offer support to each other and to the other members of the professional group.

Another factor should be taken into account when discussing the management of the suspended time. Experience shows that many recovery and rescue actions can be effective if the missing person is found within a limited span of hours, but time goes by at a different speed for rescuers and for family members. For rescuers time is spent in intense work rhythms, activating protocols and taking advantage of their rescue experience. For family members, it is a time of uncertainty that brings with it the feeling of a gradual loss given the perception of the passing of hours; there are an upheaval of the waking and sleep rhythms and a generalized disorientation. The perceived psychological time becomes interminable and suspended from the daily routines, increases the attention towards past actions and questions about actions performed before the disappearance.

The suspended time of waiting is characterized by silence. Psychologists engaged in emotional rescue know how to understand and share a silence which is a way to empathy. The non-verbal communication is aimed at sending a welcoming message. The absence of a verb was on this occasion itself verb; it was the respect of the time necessary for the elaboration, it was waiting in the hope of finding one's own life joint, it was the time needed to understand what was happening. Waiting silently together helps to understand the dynamics of family groups, to understand family relationships, to identify the most vulnerable people. Faced with an unexpected pain, there are those who isolate themselves and close in on themselves, and those who keep speaking by repeating the same anecdotes over and over. Silence is the behavior of those who place themselves in a deep listening, without interrupting those who are speaking and listening not only to words but also to what is expressed by the body language. It is empathy, accepting the pain of others without this being able to personally involve those who place themselves in a helping relationship. The emotions that are inevitably shared are experienced by the rescuer himself and this rule also applies to the psychologist. Encouraging the alternation between colleagues helps to recover the right detachment from this situation of which one becomes part. The space in the main hall acquired a meaning for the relatives: in the succession of days the same families occupied the same spaces, with composure while waiting for news. Delicacy is perhaps the adjective that best defines the approach to have in these cases. Acquiring the awareness of what happened needs time and a space protected from the intrusiveness of others and from the activities that follow one another. The task of rescuers is also to protect these spaces.

\section{Facing death}

There are several reactions resulting from the closure of research due to the finding of the body of the lost person. The missing thus becomes lost forever. The wound opens a time of intense pain, complicated by the details of the facts, often highlighted by medical-legal surveys. If on the one hand the discovery of the deceased person is the conclusion of the waiting time characterized by the anguish of uncertainty, on the other one it begins the difficult and painful path of mourning.

Supporting the news of a sudden death of a person constitutes a very intense experience both on a personal and professional level, which kindles emotions also in relation to personal experiences, to one's own experiences. It is possible that, in the face of so much pain, the psychologist himself relives painful experiences and it is therefore not recommended to expose oneself to such experiences without the support of colleagues.

The morning briefing offered the psychologists information on the state of the rescue and on the evolution of the research, on the news that had been disclosed and on the events that had occurred in the previous days. The sharing of a common operational strategy has allowed us to operate as part of a single organizational machine whose purpose was to ease the difficult moment of recognition and to start mourning the losses. The decision to allow not only the direct relatives of the missing persons, but also the extended family (uncles and friends) to flow into the hospital assembly hall, made it possible to reconstruct an affective network that could serve as a bridge between the immediacy of the news and the return of assisted people to their home. During the morning briefings of psychologists, information on those present and their degree of relationship with the missing was shared. This information was useful for identifying vulnerabilities within households: the presence of children, ${ }^{13}$ of elderly, of health problems, and the relationships between family units. All these were indicators that could help better mitigate the reactions of the frailest among the present people.

During an emergency, it is not surprising that the very intense emotional reactions can manifest themselves in a striking way or in the freezing of feelings. We assisted family members who showed photos, and talked incessantly about their relatives, as well as family members folded up in chairs in prolonged silences. The contents of the communications may be lucid or contradictory, therefore the information that is collected should be weighted, to be verified later. After a highly unexpected incident, it is normal for people, especially at first, to alternate disbelief, states of anger, and feelings of guilt. It is important to consider that these reactions are normal in front of a pathological event that destabilizes the reference points of those involved. The medicalization of emotional reactions is not always advisable, the risk of using sedatives is to deaden the grief reworking as well as pain. 
The moment of recognition is a very important space in which the family member can rejoin the body of the deceased and acquire the full awareness of death.

In tragedies, when the bodies of the dead are not found, the mourning process becomes more complex. The practice of supporting the relatives of the victims during the recognition process was entrusted to the emergency psychologists who had already followed them for a long time. It seemed natural that it should be their task to accompany the family also in this last, and often most painful, fulfillment. The relatives of the victims must be prepared for what they will face; what is going to happen must be described, as well as the spaces that will be dedicated to recognition, or the ways in which it will be done. It is now a consolidated practice to photograph significant details of the body, such as personal objects and tattoos to facilitate the task of recognition, since in case of a violent death the body of the victim may be disfigured. It is therefore important that the psychologist, before accompanying the relatives to the recognition of the body, has personally acquired the information necessary to prepare those who will be called to this task.

The practice of acquiring information and details on missing persons, experienced also in this emergency, has led to the construction of a card for the recognition of the missing, which today has been included in the Regional Plan of Maxi Health Emergencies. The card, illustrated in Figure 1, has the function of collecting as much information as possible from the relatives of missing people in order to compare them with the recovered corpses and avoid the relatives the added trauma of being exposed to many victims in an attempt to favor recognition. At the Emergency Department of the Santo Spirito Civil Hospital in

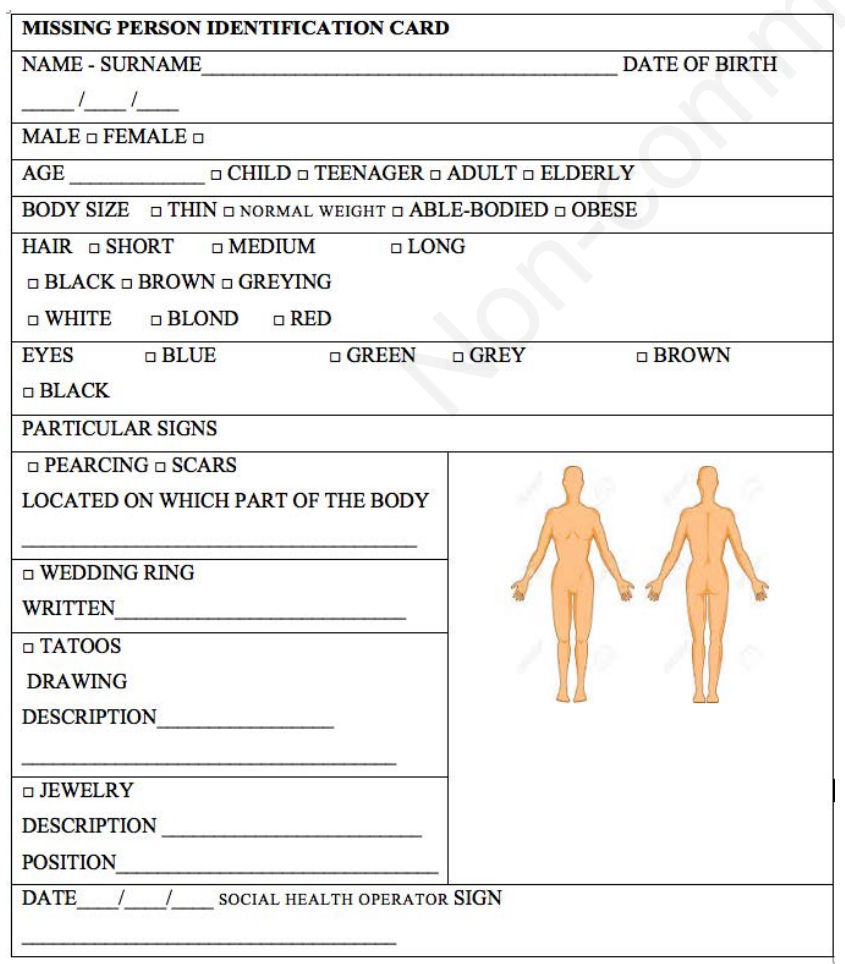

Regional Plan of Maxi Health Emergencies BURA Special $n^{\circ} 57$ of $1^{\mathrm{s}} \mathrm{June} 2018$.

Figure 1. Missing person identification card.
Pescara the victims were photographed to facilitate their identification, and the particular signs were shown to relatives that helped in the identification task. From here it was then conceived and structured a data collection form that would implement the documentation of the cases facilitating the process.

\section{Final considerations}

The Rigopiano tragedy has marked a new awareness of the preparedness and of the actions necessary to face similar situations.

The emergency protocols in Italy are sufficiently structured to deal logistically and from a health point of view with the emergencies that may affect our territory; there are experiences that are now structured in supporting victims. The experience of Rigopiano has highlighted, once again, that a machine of relief, in addition to working in favor of the primary victims of an accident, must favor the reception of those who, in various capacities, are struck by the emergency as secondary victims. The rescuers may be considered themselves as such, since they come in close contact with all the following traumatic effects of the event:

1. objective factors: traumatic stimuli, failure of the mission, human errors, serious damage to children, ${ }^{13}$ events that cause the death of colleagues, the need to make difficult choices;

2. subjective factors: tendency of the rescuers to identify themselves with the victims, need of the rescuers to keep away from the victims, lack of adequate strategies for coping with stress and/or inadequate ability to assess one's own tolerance for stress.

From the point of view of psychological operating procedures, the experience at Rigopiano forced us to reflect on the absence of manuals to draw from during emergencies. We also had to come to terms with our emotions, which at times we exposed in the same way of the people we assisted, having a similar need to be helped by our own colleagues. ${ }^{14-17}$

After January 2017, a widespread reflection involved those who, for various reasons, had experienced those days of tension, operating in the rescue of victims. As a consequence, in 2018 the Abruzzo region, writing its new plan for the maxi health emergencies, recognized psychological suffering among the needs to be met in case of disaster. In collaboration with the professionals that had been part of the psychological team on the field, the plan included important indications on the role of psychologists. In particular:

- It is established the role of the Psychosocial Manager. This officer coordinates the work of psychological teams that operate in the different contexts of the emergency, responding directly to the Regional Health Care Officer

- The need for psychologists to operate in suitable spaces is acknowledged:

In the extra-hospital setting three areas of intervention in the red zone are defined and formalized: psychological PMA, the identification area of the missing, and the zone for the protection of rescue workers.

In the intra-hospital setting four areas are defined and formalized: the area of intervention in which the relatives are received (psychological MAP), the identification area of the missing, the area of communication with the outside, and the zone for the protection of rescue workers.

It is now important to continue a dialogue between professionals in order to develop an intervention protocol structured in time 
and activities. Until now, psychologists have operated using skills and knowledge acquired in different fields of study and experiences, outside of a structured work environment. In fact, emergency psychology does not offer a specialization that can be spent in working contexts, thus making it necessary for those who work in the emergency sector that they share experiences, knowledge and know-how very often acquired with practical experience in the field.

Sharing is therefore the new challenge for the psychology of emergency. This might lead to organize training at a regional level and to develop an intervention methodology that goes beyond the boundaries of associations and guarantees a uniform professional assistance. Moreover, psychological support should not be limited just to overcoming the trauma of individuals, but should grow to take charge also of the communities hit by calamities, offering them a resilient push to the reconstruction of social networks.

\section{Conclusions}

In conclusion, it must always be remembered that people at the center of an emergency, beyond physical trauma, carry a psychological trauma. We must also consider that, in addition to the victims directly struck by an accident or a disaster, there are their families and their communities, which, too often silently, try to overcome their difficult experiences. Psychologists are called to reconstruct the plots of these lives and to support the task of those who are committed to saving lives.

\section{References}

1. Jacobs GA. Community-based psychological first aid: a practical guide to helping individuals and communities during difficult time. Oxford: Elsevier; 2016.

2. Giel R. Psychosocial process in disaster. Int J Ment Health 1990;19:7-20.

3. Green BL. Evaluating the effects of disaster "Psychological Assessment". J Consult Clin Psychol 1991;538-46.

4. Watson P, Hamblen J. Assisting individuals and communities after natural disasters and community traumas. In: S.N. Gold
(Ed.-in-Chief). APA handbook of trauma psychology (Vol. 1). Washington, DC: American Psychological Association; 2017.

5. Pernille T. Strengthening resilience. A global selection of psychosocial interventions, for Red Cross and Red Crescent Societies, IFRC Reference Centre for Psychosocial Support. Copenhagen; 2014.

6. Goldmann E, Galea S. Mental health consequences of disasters. Annu Rev Public Health 2014;35:169-83.

7. Ministerial Decree 13th February 2001: Broad criteria for health relief in disasters. Gazzetta Ufficiale n. 81, 6 april 2001.

8. Persoff J, Ornoff D, Little C. The role of hospital medicine in emergency preparedness: a framework for hospitalist leadership in disaster preparedness, response, and recovery. J Hosp Med 2018;13:713-8.

9. Becker TK, Hansoti B, Bartels S, et al. Global Emergency Medicine Literature Review (GEMLR) Group. Acad Emerg Med 2017;24:1150-60.

10. Jensen J, Thompson S. The Incident Command System: a literature review. Disasters 2016;40:158-82.

11. Pernille H. A Guide to Psychological First Aid for Red Cross and Red Crescent Societies, IFRC Reference Centre for Psychosocial Support. Copenhagen; 2018.

12. Guilaran J, de Terte I, Kaniasty K, et al. Psychological outcomes in disaster responders: a systematic review and metaanalysis on the effect of social support. Int J Disaster Risk Sci 2018;9:344-58.

13. Cohen RE. Intervention programs for children. In: M. Lystand, Ed., Mental health response to mass emergencies: Theory and practice. New York: Brunner/ Mazel; 1998.

14. Armstrong KR, O'Callahan WE, Mermar CR. Debriefing Red Cross Disaster personnel: The multiple stressor debriefing model. J Trauma Stress 1991;4:581-3.

15. Hiley-Yuong B, Gerrity E. Critical incident stress debriefing (CISD): Value and limitations in disaster response. NCP Clinical Quarterly 1994;4:17-9.

16. Riggio RE, Riggio HR, Salinas C, Cole EJ. The Role of Social and Emotional Communication Skills in Leader Emergence and Effectiveness. Group Dyn Theory Res Pract 2003;7:83103.

17. Prati G, Pietrantoni L. The relation of perceived and received social support to mental health among first responders: A metaanalytic review. J Comm Psychol 2010;38:403-17 